\title{
Persistently high prevalence of primary resistance and multidrug resistance of tuberculosis in Heilongjiang Province, China
}

\author{
Di Li ${ }^{1,2+}$, Jing-Li Wang ${ }^{1+}$, Bin-Ying Ji ${ }^{4}$, Jia-Yi Cui ${ }^{1}$, Xin-Ling Pan ${ }^{1}$, Chang-Long Fan ${ }^{4}$, \\ Chang-Xia Shao', Li-Na Zhao", Yuan-Ping Ma', Liu-Zhuo Zhang ${ }^{5}$, Chun-Lei Zhang ${ }^{4}$, Cai-Bo Dong1', \\ Toshio Hattori ${ }^{6}$ and Hong Ling ${ }^{1,2,3^{*}}$
}

\begin{abstract}
Background: The spread of multidrug-resistant tuberculosis (MDR-TB) Mycobacterium tuberculosis (M. tuberculosis) strains has been a big challenge to the TB control and prevention in China. Knowledge about patterns of drug resistance in TB high-burden areas of China is crucial to develop appropriate control strategies. We conducted a comprehensive investigation of the resistance pattern of M. tuberculosis in Heilongjiang Province.

Methods: 1427 M. tuberculosis clinical strains were isolated from pulmonary TB patients hospitalized between 2007 and 2012. The susceptibility of the isolates to the first-line anti-TB drugs and the resistance of MDR M. tuberculosis to fluoroquinolones were examined. We also performed a statistical analysis to identify the correlated risk factors for high burden of MDR-TB.

Results: The global resistance rates of 2007-2012 to the first-line drugs and MDR were 57.0 and $22.8 \%$, respectively. Notably, the primary MDR-TB and pan-resistance rates were as high as 13.6 and $5.0 \%$, respectively. Of MDR M. tuberculosis isolates (2009), approximately $13 \%$ were not susceptible to any of the fluoroquinolones tested. Being age of 35 to 54, high re-treatment proportion, the presence of cavity lesion, and high proportion of shorter hospitalization are correlated with the development of MDR-TB.

Conclusions: The high prevalence of drug resistant, MDR-TB, and fluoroquinolone-resistant MDR-TB is a big concern for TB control. More importantly, in order to control the development of MDR-TB effectively, we need to pay more attention to the primary resistance. Targeting reducing the prevalence of the risk factors may lead to better TB control in China.
\end{abstract}

Keywords: Mycobacterium tuberculosis, Primary resistance, Multi-drug resistance, Risk factors

\section{Background}

The spread of drug-resistant and multidrug-resistant (MDR) tuberculosis (TB) is a severe global health issue. In recent years, both the incidence and prevalence of TB in China have steadily declined [1]. The World Health Organization (WHO) indicates that the MDR rates in China $(5.7 \%$ in new cases and $26 \%$ in previously treated cases, respectively) have become lower than in other

\footnotetext{
* Correspondence: lingh@ems.hrbmu.edu.cn

'Equal contributors

'Department of Microbiology; Wu Lien-Teh Institute, Harbin Medical University, Harbin, China

${ }^{2}$ Heilongjiang Provincial Key Laboratory of Infection and Immunity, Harbin, China Full list of author information is available at the end of the article
}

countries among the 27 high MDR-TB burden countries. However, the rates remain higher than the global average rates of 3.6 and $20 \%$ for new and previously treated cases, respectively. China is ranked second in the number of MDR-TB cases after India because of China's large population. Moreover, the global extensively drugresistant (XDR)-TB prevalence is increasing, with $9.6 \%$ MDR-TB cases in 2012 compared to $5.4 \%$ in 2007. We estimate an increasing XDR-TB prevalence in China, with $8 \%$ from MDR-TB cases, because this rate was higher than the worldwide level in 2007 [1]. The global spread of XDR-TB has led to new challenges for the prevention and control of tuberculosis. 
The epidemic trends of drug resistant-TB in different geographic areas of China vary greatly, and recently, the spread of TB in China has been caused by active domestic migration, resulting in a significant public health issue. Heilongjiang Province is located in Northeast China, a region in which the prevalence rates of both $\mathrm{TB}$ and drugresistant TB are higher than the average in China [2-4]. The rates of any resistance to first-line drugs were 38.9 and $36.2 \%$ for new cases and 70.9 and $67.7 \%$ for retreated cases in Heilongiiang Province in 2002 and 2004, respectively, based on data from population-based drug resistance investigations [2-4].

It is important to identify the causes for the high prevalence of drug-resistance TB. The risks associated with drug resistant- and MDR-TB include a history of retreatment using anti-mycobacterial drugs, having cavities, Beijing genotype epidemic, low socio-economic status, age and DOTS (directly observed treatment short course) implementation. However, the factors vary depending on the study $[1,5-8]$.

Moreover, we would like to determine whether the prevalence of fluoroquinolone resistant TB in Heilongjiang Province is also high. MDR-TB is a risk factor for the resistance of $M$. tuberculosis to fluoroquinolones $[9,10]$. In China, fluoroquinolones are the most frequently used antibiotics for the treatment of a variety of infectious diseases [11-14]. XDR-TB arises when MDR-TB strains acquire resistance to any fluoroquinolone, and previous treatment using second-line drugs, including fluoroquinolones, is a significant risk factor for XDR-TB $[15,16]$.

In the present study, we analyzed the resistance trends of M. tuberculosis clinical isolates from 2007 to 2012 to first-line drugs and fluoroquinolones and the risk factors in Heilongjiang Province, an area with the highest tuberculosis disease and drug resistance burden in China. Elucidating the locally significant risk factors for the high prevalence of TB is important to control the spread of $\mathrm{TB}$ and drug resistant $\mathrm{TB}$ in this area and other regions of China.

\section{Methods}

\section{Mycobacterial specimen and data collection}

All the data we collected including patient medical records are available in Harbin Chest Hospital. A total of 1427 isolates from 1427 patients, who were diagnosed with TB at the Harbin Chest Hospital from May 2007 and July 2012 and from various regions of Heilongjiang Province, were included. The patients were HIV-1 negative. The patient information of gender, age, TB treatment history, and the presence of cavity on chest radiographs was from hospital medical records. Ethical clearance and approval for this study was provided by the Institutional Research Board of the University of Harbin Medical University (Ethics Reference No.: HMUIRB20160001).

\section{Mycobacterial culture and drug susceptibility testing (DST) to first-line drugs}

Mycobacterial cultures were obtained from clinical specimens after incubation in a BACTEC Mycobacterium Growth Indicator Tube (MGIT) 960 Automated System (BD Diagnostic Systems, Franklin Lakes, NJ, USA). Primary identification was performed using the Ziehl-Neelsen staining method and microscopy. The tests of inhibition by P-nitrobenzoic acid and 2-Thiophenecarboxylic acid hydrazide were used to differentiate $M$. tuberculosis from other Mycobacterium spp. DST to first-line drugs was performed using MGIT 960 SIRE Kit. Strict controls including growth control (M. tuberculosis H37Rv growth control in drugfree tube) and the negative control ( $\mathrm{H} 37 \mathrm{Rv}$ in the presence of each drug) were used according to the instruction of MGIT 960 SIRE Kit.

The resistance of MDR $M$. tuberculosis to fluoroquinolones Resistance of some MDR isolates from 2009 to 2012 to FQs was examined in the Hospital as clinical departments suggested. The susceptibility of the MDR strains isolated in 2009 ( $n=52$, recovered from the frozen stock) to FQs including levofloxacin (Sigma), sparfloxacin (Sigma), moxifloxacin (Hubei Saibo Pharmaceutical company, Wuhan, China), gemifloxacin (Hubei Saibo Pharmaceutical company, Wuhan, China), and gatifloxacin (National Institutes for Food and Drug Control, Beijing, China) was examined by minimun inhibition concentrations (MIC) assay. The test was performed using the standard microdilution method as described previously [17]. Initial concentration was $16 \mathrm{mg} / \mathrm{L}$ for both levofloxacin and sparfloxacin, and $8 \mathrm{mg} / \mathrm{L}$ for moxifloxacin, gemifloxacin, and gatifloxacin. Seven serial two-fold dilutions of each drug in Middlebrook 7H9 were performed [18-20]. Each well was inoculated with a 0.03 McFarland mycobacterial suspension (final inoculums was $2.85 \times 10^{5} \mathrm{CFU} / \mathrm{mL}$ ). Two wells without drugs were inoculated with $\mathrm{H} 37 \mathrm{Rv}$ as a growth control. All peripheral wells of the plates were filled with sterile distilled water. The plates were incubated at $37^{\circ} \mathrm{C}$ for 16 to 18 days. For each drug, the lowest concentration that displayed no visible turbidity was defined as the MIC. Three independent assays were performed.

The susceptibility of MDR isolates from 2010 to 2012 to levofloxacin was determined by using absolute concentration method on solid Lowenstein-Jensen medium (Hangzhou Genesis Biodetection \& Biocontrol Ltd) upon National Guideline. The concentration of $2 \mathrm{mg} / \mathrm{L}$ was set as the breakpoint [21].

DNA extraction, PCR-based identification of $M$. tuberculosis, and Beijing genotype determination

Bacterial DNA was extracted after the inactivation of mycobacterial isolates in $70 \%$ ethanol for $2 \mathrm{~h}$ [22]. DNA was extracted using lysozyme and the phenol-chloroform 
method [23]. Molecular identification of M. tuberculosis with the PCR amplifications of the genes including $16 \mathrm{~S}$ rRNA, Rv0577, Rv2073c, and Rv3120 was performed as previously described $[24,25]$. M. tuberculosis strains have positive amplifications of the four genes. M. microti and M. tuberculosis $\mathrm{H} 37 \mathrm{Rv}$ was used as reference strains.

To identify the Beijing genotype of the isolates, RD105 deletion PCR was performed as described previously [24].

\section{Statistical analysis}

The odds ratio was used to evaluate the univariate and multivariate risk factors associated with MDR-TB and drug resistant- (not MDR) TB. The following variables were included in the analysis: the patient's gender, age, TB treatment history, presence of a cavity on chest radiograph, hospitalization time and Beijing genotype. All of the variables were initially included in the model, and the forward method was used to select the final variables. The statistical interaction between relevant variables was assessed. All statistical analyses were performed using SPSS version 17.0 (SPSS Inc., Chicago, IL, USA).

\section{Results}

Sample and data collection

Of the 1427 patients with pulmonary tuberculosis identified from May 2007 through July 2012, 918 (64.3 \%) and $509(35.7 \%)$ were new and re-treated cases, respectively (Table 1). The medical records were available for all cases. Chest radiographs indicating the presence or absence of cavity were available for 1254 cases. Three hundred isolates from May 2007 through September 2009 were re-confirmed by PCR-based identification and were further examined to identify the Beijing family genotype and phylogeny based on the VNTR genotypes. Fifty two re-cultured available MDR $M$. tuberculosis isolates from 2009 were examined for sensitivity to fluoroquinolones (MIC). The susceptibility of MDR isolates from 2010 to 2012 to levofloxacin was available from routine clinical drug resistance examinations in hospital.

\section{High prevalence of drug-resistant TB in Heilongjiang Province from 2007 to 2012}

Overall, the average resistance rate of $\mathrm{TB}$ to any firstline drugs from 2007 to 2012 was $57.0 \%$ (Table 1). The drug resistance rate among new case and re-treated case

Table 1 Susceptibility of the $M$.tuberculosis isolated in Heilongjiang Province to the first-line anti-tuberculosis drugs

\begin{tabular}{|c|c|c|c|c|c|c|c|c|c|c|c|c|c|c|}
\hline \multirow[t]{2}{*}{ Characteristics } & \multicolumn{2}{|l|}{2007} & \multicolumn{2}{|c|}{2008} & \multicolumn{2}{|l|}{2009} & \multicolumn{2}{|c|}{2010} & \multicolumn{2}{|c|}{2011} & \multicolumn{2}{|l|}{2012} & \multicolumn{2}{|l|}{ Total } \\
\hline & No. & $\%$ & No. & $\%$ & No. & $\%$ & No. & $\%$ & No. & $\%$ & No. & $\%$ & No. & $\%$ \\
\hline All & 198 & & 348 & & 275 & & 204 & & 205 & & 197 & & 1427 & \\
\hline Any drug resistance & 110 & 55.6 & 209 & 60.1 & 189 & 68.7 & 121 & 59.3 & 89 & 43.4 & 96 & 48.7 & 814 & 57.0 \\
\hline RPF or INH ${ }^{a, b}$ & 42 & 21.2 & 77 & 22.1 & 72 & 26.2 & 55 & 27.0 & 42 & 20.5 & 40 & 20.3 & 328 & 23.0 \\
\hline SM & 72 & 36.4 & 152 & 43.7 & 125 & 45.5 & 86 & 42.2 & 63 & 30.7 & 63 & 32.0 & 561 & 39.3 \\
\hline EMB & 47 & 23.7 & 74 & 21.3 & 75 & 27.3 & 16 & 7.8 & 17 & 8.3 & 18 & 9.1 & 247 & 17.3 \\
\hline MDR & 44 & 22.2 & 90 & 25.9 & 81 & 29.5 & 39 & 19.1 & 33 & 16.1 & 38 & 19.3 & 325 & 22.8 \\
\hline Pan-resistance ${ }^{c}$ & 21 & 10.6 & 49 & 14.1 & 37 & 13.5 & 10 & 4.9 & 12 & 5.9 & 11 & 5.6 & 140 & 9.8 \\
\hline From new cases & 126 & & 218 & & 171 & & 143 & & 136 & & 124 & & 918 & \\
\hline Any drug resistance & 62 & 49.2 & 113 & 51.8 & 103 & 60.2 & 74 & 51.7 & 46 & 33.8 & 49 & 39.5 & 447 & 48.7 \\
\hline RPF or INH & 24 & 19.0 & 43 & 19.7 & 46 & 26.9 & 37 & 25.9 & 24 & 17.6 & 23 & 18.5 & 197 & 21.5 \\
\hline SM & 42 & 33.3 & 84 & 38.5 & 67 & 39.2 & 53 & 37.1 & 32 & 23.5 & 31 & 25.0 & 309 & 33.7 \\
\hline EMB & 19 & 15.1 & 32 & 14.7 & 38 & 22.2 & 9 & 6.3 & 6 & 4.4 & 5 & 4.0 & 109 & 11.9 \\
\hline MDR & 20 & 15.9 & 36 & 16.5 & 29 & 17.0 & 18 & 12.6 & 11 & 8.1 & 11 & 8.9 & 125 & 13.6 \\
\hline Pan-resistance & 5 & 4.0 & 17 & 7.8 & 14 & 8.2 & 4 & 2.8 & 4 & 2.9 & 2 & 1.6 & 46 & 5.0 \\
\hline From re-treated cases & 72 & & 130 & & 104 & & 61 & & 69 & & 73 & & 509 & \\
\hline Any drug resistance & 48 & 66.7 & 96 & 73.8 & 86 & 82.7 & 47 & 77.0 & 43 & 62.3 & 47 & 64.4 & 367 & 72.1 \\
\hline RPF or INH & 18 & 25.0 & 34 & 26.2 & 26 & 25.0 & 18 & 29.5 & 18 & 26.1 & 17 & 23.3 & 131 & 25.7 \\
\hline SM & 30 & 41.7 & 68 & 52.3 & 58 & 55.8 & 33 & 54.1 & 31 & 44.9 & 32 & 43.8 & 252 & 49.5 \\
\hline EMB & 28 & 38.9 & 42 & 32.3 & 37 & 35.6 & 7 & 11.5 & 11 & 15.9 & 13 & 17.8 & 138 & 27.1 \\
\hline MDR & 24 & 33.3 & 54 & 41.5 & 52 & 50.0 & 21 & 34.4 & 22 & 31.9 & 27 & 37.0 & 200 & 39.3 \\
\hline Pan-resistance & 16 & 22.2 & 32 & 24.6 & 23 & 22.1 & 6 & 9.8 & 8 & 11.6 & 9 & 12.3 & 94 & 18.5 \\
\hline
\end{tabular}

a INH isoniazid, RFP rifampicin, MDR multidrug-resistance, SM streptomycin, EMB ethambutol

${ }^{b}$ The isolates were resistant to RPF or INH but not both

${ }^{\mathrm{C}}$ The isolates were resistant to all the four first-line drug 
were 48.7 and $72.1 \%$ respectively. The MDR-TB rate was $22.8 \%$. Of the MDR cases, 13.6 and $39.3 \%$ were new and re-treated cases, respectively. The highest prevalence of resistance to the first-line drugs and MDR from either the new or retreated cases occurred in 2009 and the lowest occurred in 2011 (Table 1).

There was also a high prevalence of primary resistance (Table 1). Of the cases resistant to any first-line drug and MDR-TB cases, approximately 55 and $39 \%$, respectively, were new cases. From 2007 to 2012, the yearly resistance to any first-line drug among new cases, was 49.2, 51.8, $60.2,51.7,33.8$, and $39.5 \%$, respectively. The MDR rates were $15.9,16.5,17.0,12.6,8.1$, and $8.9 \%$, respectively. Notably, $5.0 \%$ of the pan-resistant cases (resistant to all 4 first-line drugs) were detected in the new cases.

Importantly, the resistance of the re-treated cases was significant (Table 1). Of the cases resistant to any of the first-line drugs and MDR-TB cases, approximately 45.1 and $61.5 \%$, respectively, were re-treated cases. From 2007 to 2012, the resistance rates of the re-treated cases to any first-line drug were $66.7,73.8,82.7,77.0,62.3$, and $64.4 \%$, respectively. The annual rates of MDR were $33.3,41.5,50.0,34.4,31.9$, and $37 \%$, respectively. Of the re-treated cases, $18.5 \%$ were pan-resistant.

\section{The resistance of MDR M. tuberculosis isolates to fluoroquinolones}

Because the resistance rate to the first-line drugs in 2009 was the highest, resistance to fluoroquinolones of some MDR isolates (MIC) was conducted upon suggestion of clinical departments in strengthening the efficacy of treatment (Table 2). The critical concentration for levofloxacin is $2.0 \mathrm{mg} / \mathrm{L}$, as per the World Health Organization, and was used to categorize the isolates as susceptible or nonsusceptible. Because there are no recommended critical concentrations for sparfloxacin, moxifloxacin, gatifloxacin and gemifloxacin, we further analyzed the proportion of isolates with MICs equal to and higher than $2 \mathrm{mg} / \mathrm{L}$, based on the serum concentrations of the drugs [20], and they were defined as non-sensitive to a drug.

Of the MDR isolates, 36.5, 26.9, 38.4, and $40.4 \%$ were not susceptible to sparfloxacin, moxifloxacin, gemifloxacin and gatifloxacin, respectively. We also found that there were high proportions of MDR $M$. tuberculosis isolates resistant to the fluoroquinolones (Table 3). Approximately $21 \%$ of the isolates were not susceptible to levofloxacin and moxifloxacin. The resistance rates to the 3rd and 4th generation of fluoroquinolones were $15.4-17.3 \%$ and $13.5-15.4 \%$, respectively. Notably, $13.5 \%$ of the isolates were not susceptible to 5 fluoroquinolones.

We also analyzed the results of the susceptibility test of $M$. tuberculosis to levofloxacin that was available since 2010. We found that $22.6 \%(7 / 31), 27.6 \%(8 / 29)$, and $25 \%$ (9/36) MDR isolates from 2010, 2011, and 2012, respectively, were resistant to levofloxacin.

\section{Factors linked to drug-resistant TB}

The risk factors associated with drug resistance were analyzed (Table 4). We found that an age of 35 to 54 (OR 2.5, $95 \%$ CI 1.8-3.4), re-treatment history (OR 5.3, $95 \%$ CI 4.0-7.1), the presence of cavity (OR 1.8, $95 \%$ CI 1.32.5), shorter hospitalization (OR 6.4, $95 \%$ CI 4.8-8.7), and infection with the Beijing genotype (OR 4.3, $95 \%$ CI 1.2-15.1) were high-risk factors for the development of MDR-TB based on the univariate analysis. The multivariate analysis further confirmed these findings, except that the Beijing genotype was not included in the final logistic-regression models [26].

Furthermore, MDR-TB cases had a higher frequency $(61.5 \%)$ of re-treatment compared to the drug resistantTB cases (34.2\%). Of the MDR cases, $60.3 \%$ had non-

Table 2 Susceptibilities of MDR M. tuberculosis isolates of 2009 to fluoroquinolones ${ }^{a}$

\begin{tabular}{|c|c|c|c|c|c|c|c|c|c|c|}
\hline \multirow[t]{2}{*}{ MIC (mg/L) } & \multicolumn{2}{|c|}{ Levofloxacin } & \multicolumn{2}{|c|}{ Moxifloxacin } & \multicolumn{2}{|c|}{ Sparfloxacin } & \multicolumn{2}{|c|}{ Gemifloxacin } & \multicolumn{2}{|c|}{ Gatifloxacin } \\
\hline & No. & $\%$ & No. & $\%$ & No. & $\%$ & No. & $\%$ & No. & $\%$ \\
\hline$\leq 0.125$ & & & & & & & 2 & 3.8 & 1 & 1.9 \\
\hline$\leq 0.25$ & 7 & 13.4 & 5 & 9.6 & 14 & 26.9 & 7 & 13.5 & 8 & 15.4 \\
\hline 0.50 & 10 & 19.2 & 18 & 34.6 & 9 & 17.3 & 14 & 26.9 & 15 & 28.8 \\
\hline $1 \mathrm{mg} / \mathrm{L}$ & 15 & 28.8 & 15 & 28.8 & 10 & 19.2 & 9 & 17.3 & 7 & 13.5 \\
\hline 2.00 & 8 & 15.4 & 9 & 17.3 & 8 & 15.4 & 14 & 26.9 & 12 & 23.1 \\
\hline 4.00 & 8 & 15.4 & 3 & 5.8 & 7 & 13.5 & 6 & 11.5 & 7 & 13.5 \\
\hline 8.00 & 4 & 7.7 & 2 & 3.8 & 3 & 5.8 & & & 2 & 3.8 \\
\hline 16.00 & & & & & 1 & 1.9 & & & & \\
\hline $\mathrm{MIC} \geq 2 \mathrm{mg} / \mathrm{L}$ & 20 & 38.5 & 14 & 26.9 & 19 & 36.5 & 20 & 38.4 & 21 & 40.4 \\
\hline $\mathrm{MIC} 50^{\mathrm{b}}$ & 1.0 & & 1.0 & & 1.0 & & 1.0 & & 1.0 & \\
\hline
\end{tabular}

${ }^{\mathrm{a}} \mathrm{MDR}$ multidrug resistance, $M I C$ minimum inhibitory concentration

${ }^{\mathrm{b}} \mathrm{MIC}_{50}$, minimum inhibitory concentration required to inhibit the growth of $50 \%$ of examining clinical isolates 
Table 3 Multiple resistance of MDR-Mtb isolates of 2009 to FQs $(n=52)^{a}$

\begin{tabular}{|c|c|c|c|c|}
\hline \multirow[t]{2}{*}{ Category } & \multicolumn{2}{|c|}{$\mathrm{MIC}<2 \mathrm{mg} / \mathrm{L}$} & \multicolumn{2}{|c|}{$\mathrm{MIC} \geq 2 \mathrm{mg} / \mathrm{L}$} \\
\hline & No. & $\%$ & No. & $\%$ \\
\hline$L f x+M f x$ & 29 & 55.8 & 11 & 21.2 \\
\hline$L f x+M f x+G f x$ & 24 & 46.2 & 8 & 15.4 \\
\hline$L f x+M f x+G m x$ & 27 & 51.9 & 9 & 17.3 \\
\hline$L f x+M f x+S f x$ & 26 & 50.0 & 8 & 15.4 \\
\hline$L f x+M f x+S f x+G m x$ & 25 & 48.1 & 8 & 15.4 \\
\hline$L f x+M f x+G f x+G m x$ & 24 & 46.2 & 7 & 13.5 \\
\hline$L f x+M f x+G f x+S f x$ & 24 & 46.2 & 7 & 13.5 \\
\hline$L f x+M f x+G f x+S f x+G m x$ & 24 & 46.2 & 7 & 13.5 \\
\hline
\end{tabular}

${ }^{\mathrm{a} M D R}$ multidrug resistance, $M t b$ M. tuberculosis, FQs fluoroquinolones, MIC minimum inhibitory concentration, Lfx levofloxacin, Sfx sparfloxacin, $M f x$ moxifloxacin, Gmx gemifloxacin, Gfx gatifloxacin

standard hospitalization history compared to $19 \%$ of the drug resistant-TB cases.

Overall, the proportion of cases with cavity, aged 35 to 54 , and re-treatment history were $64.8,41.1$, and $35.7 \%$, respectively, in the TB patients from the study area
(Table 4). In this region, the Beijing genotype prevalence was $89.7 \%$. However, the proportion of cases with nonstandard hospitalization was $28.5 \%$.

\section{Discussion}

Heilongjiang Province is an area of China with high burdens of drug resistant- and MDR-TB [2-4]. However, the reported resistance rates were based on data from the China CDC system. In China, the CDC system generally takes care of outpatients, whereas TB hospitals accept either outpatients or inpatients. The complicated TB cases are usually admitted to TB hospitals. Nationwide studies have shown that first line drug resistance and MDR rates are different among different geographical areas and patient population [1, 10, 27-46] (Table 5). The resistance rates of clinical isolates from 2007 to 2014 to first-line drugs were higher in hospitalized patients $[10,31,34,38,39,42-44]$. On the other hand, surveys based on wide range of TB cases (including outpatients and inpatients) exhibited comparatively lower resistance rates $[29,30,32,37]$. The MDR prevalence showed similar trend [10, 29-32, 34-46].

Table 4 Univariate and multivariate analysis of risk factors for drug-resistant (not MDR) and MDR TB ${ }^{a}$

\begin{tabular}{|c|c|c|c|c|c|c|c|c|}
\hline \multirow[t]{2}{*}{ Characteristic } & \multirow[b]{2}{*}{$\begin{array}{l}\text { Total } \\
(n=1427)\end{array}$} & \multicolumn{3}{|c|}{ No. (\%) of patients } & \multicolumn{2}{|c|}{$\begin{array}{l}\text { Drug-resistant vs. Pan-sensitive } \\
\text { OR }(95 \% \mathrm{Cl})\end{array}$} & \multicolumn{2}{|c|}{$\begin{array}{l}\text { MDR vs. Pan-sensitive } \\
\text { OR ( } 95 \% \mathrm{Cl})\end{array}$} \\
\hline & & $\begin{array}{l}\text { Pan-sensitive } \\
(n=613)\end{array}$ & $\begin{array}{l}\text { Drug-resistant } \\
(n=489)\end{array}$ & $\begin{array}{l}\text { MDR } \\
(n=325)\end{array}$ & $\begin{array}{l}\text { Univariate } \\
\text { analysis }\end{array}$ & $\begin{array}{l}\text { Multivariate } \\
\text { analysis }\end{array}$ & $\begin{array}{l}\text { Univariate } \\
\text { analysis }\end{array}$ & $\begin{array}{l}\text { Multivariate } \\
\text { analysis }\end{array}$ \\
\hline \multicolumn{9}{|l|}{ Sex } \\
\hline Male & $994(69.7)$ & $425(69.3)$ & $343(70.1)$ & $226(69.5)$ & Referent & NA & Referent & NA \\
\hline Female & 433(30.3) & $188(30.7)$ & 146(29.9) & $99(30.5)$ & $1.0(0.8-1.3)$ & NA & $1.0(0.7-1.4)$ & NA \\
\hline \multicolumn{9}{|l|}{ Age group, y } \\
\hline $7-34$ & $469(32.9)$ & 234(38.2) & 152(31.1) & $83(25.5)$ & Referent & Referent & Referent & Referent \\
\hline $35-54$ & $587(41.1)$ & $198(32.3)$ & $215(44.0)$ & $174(53.5)$ & $1.7(1.3-2.2)$ & $1.5(1.1-2.0)$ & $2.5(1.8-3.4)$ & $1.7(1.2-2.5)$ \\
\hline$\geq 55$ & $371(26.0)$ & $181(29.5)$ & $122(24.9)$ & $68(20.9)$ & $1.0(0.8-1.4)$ & $0.9(0.7-1.2)$ & $1.0(0.7,1.5)$ & $0.6(0.4-0.9)$ \\
\hline \multicolumn{9}{|c|}{ History of TB treatment } \\
\hline No & $918(64.1)$ & $471(76.8)$ & $322(65.8)$ & $125(38.5)$ & Referent & Referent & Referent & Reference \\
\hline Yes & $509(35.7)$ & 142(23.2) & 167(34.2) & $200(61.5)$ & $1.7(1.3-2.2)$ & $1.7(1.2-2.1)$ & $5.3(4.0-7.1)$ & $3.6(2.6-5.0)$ \\
\hline \multicolumn{9}{|c|}{ Cavity visible on radiograph ${ }^{b}$} \\
\hline No & $442(35.2)$ & $222(39.8)$ & 147(34.9) & $73(26.5)$ & Referent & Referent & Referent & Referent \\
\hline Yes & $812(64.8)$ & $336(60.2)$ & $274(65.1)$ & $202(73.5)$ & $1.2(0.9-1.6)$ & $1.2(0.9-1.6)$ & $1.8(1.3-2.5)$ & $1.6(1.0-2.2)$ \\
\hline \multicolumn{9}{|c|}{ With standard hospitalization } \\
\hline Yes & $1021(71.5)$ & $496(80.9)$ & $396(81.0)$ & $129(39.7)$ & Referent & NA & Referent & Referent \\
\hline $\mathrm{No}^{\mathrm{c}}$ & $406(28.5)$ & $117(19.1)$ & 93(19.0) & $196(60.3)$ & $1.0(0.7-1.3)$ & NA & $6.4(4.8-8.7)$ & $5.0(3.6-7.0)$ \\
\hline \multicolumn{9}{|c|}{ Beijing genotype $^{d}$} \\
\hline No & $31(10.3)$ & $17(14.8)$ & $11(10.2)$ & $3(3.9)$ & Referent & NA & Referent & NA \\
\hline Yes & 269(89.7) & $98(85.2)$ & $97(89.8)$ & $74(96.1)$ & $1.5(0.7-3.4)$ & NA & $4.3(1.2-15.1)$ & NA \\
\hline
\end{tabular}

${ }^{a} M D R$ multidrug resistance, $O R$ odds ratio, $C l$ confidence interval, NA not applicable (These variables were not included in the multivariate logistic-regression models)

${ }^{\mathrm{b}}$ The radiograph results were available with 1254 patients during the period 2007 through 2012

'The hospitalization days of the cases were shorter than the required by the National guidelines: $21-28,28-35$ and $42-56$ days for non-MDR new, non-MDR retreated and MDR cases, respectively [26]

dBeijing genotype analysis with 300 isolates from 2007 to 2009 was available 
Table 5 The resistance of Mtb isolated in China to first-line drugs and FQs ${ }^{a}$

\begin{tabular}{|c|c|c|c|c|c|c|c|c|c|}
\hline \multirow[t]{2}{*}{ Location (Ref.) } & \multirow{2}{*}{$\begin{array}{l}\text { Sampling } \\
\text { Year }\end{array}$} & \multirow{2}{*}{$\begin{array}{l}\text { Sampling } \\
\text { source }^{b}\end{array}$} & \multirow{2}{*}{$\begin{array}{l}\text { Sample } \\
\text { size }\end{array}$} & \multicolumn{2}{|c|}{ First-line Resistance (\%) } & \multicolumn{2}{|c|}{ MDR rate (\%) } & \multicolumn{2}{|c|}{ FQ Resistance (\%) } \\
\hline & & & & Total & New & Total & New & Total & MDR \\
\hline \multirow[t]{3}{*}{ China $[1,27,28]$} & 2007 & Survey & 3929 & 38.3 & 34.2 & 10.2 & 5.7 & & \\
\hline & $2007-2008$ & Survey & 3634 & & & 9.9 & & 4.0 & \\
\hline & $2005-2012$ & Hospital & 450 & 54.4 & 44.7 & 25.8 & 14.5 & & \\
\hline Jilin [29] & 2008-2011 & Survey & 1772 & 40.1 & 33.1 & 13.5 & 8.60 & & \\
\hline Ningxia [30] & 2013-2014 & Survey & 665 & 26.6 & 20.2 & 7.8 & & & \\
\hline Chongqing [31] & 2009-2013 & Hospital & 2271 & 61.9 & 52.9 & 26.3 & 16.7 & & \\
\hline Lianyungang, Jiangsu [32] & $2011-2012$ & Survey & 1012 & 30.4 & 23.4 & 8.7 & 4.2 & & \\
\hline Kaohsiung, Taiwan [33] & $2000-2008$ & Hospital & 421 & 15.2 & & 2.1 & & & \\
\hline Beijing [34] & $2007-2009$ & Hospital & 967 & 70.1 & 60.9 & 19.4 & 14.9 & $\underline{27.1}$ & 35.1 \\
\hline Xinjiang $[35,36]$ & 2009-2013 & Hospital & 410 & & & 13.2 & 12.9 & & \\
\hline \multirow[t]{3}{*}{ Heilongjiang (present) } & $2007-2012$ & Hospital & 1427 & 57.0 & 48.7 & 22.8 & 13.6 & & \\
\hline & $2007-2009$ & Hospital & 821 & 61.9 & 60.0 & 26.2 & 16.5 & & \\
\hline & $2010-2012$ & Hospital & 606 & 50.5 & 41.9 & 18.2 & 9.9 & 10.4 & 25.0 \\
\hline Kunming, Yunnan [37] & $2008-2009$ & CDC & 279 & 31.5 & & 9.3 & & & \\
\hline Hunan [38] & 2009-2010 & Hospital & 171 & 40.9 & 19.6 & 25.2 & 6.2 & 10.5 & \\
\hline Xuzhou, Jiangsu [39] & $2011-2012$ & Hospital & 287 & 16.0 & & 3.14 & & & \\
\hline Zhejiang [40] & $2011-2012$ & Hospital & 1363 & & & 16.7 & & & \\
\hline Rural China [10] & 2008 & Hospital & 380 & 31.1 & 25.9 & 11.3 & 9.0 & $\underline{10.8}$ & $\underline{23.3}$ \\
\hline Jiangxi [41] & 2010-2011 & Hospital & 804 & & & 19.8 & & & \\
\hline Northeast China [42] & 2010-2011 & Hospital & 205 & 26.3 & 22.0 & 6.8 & 3.0 & & \\
\hline Anhui [43] & 2010-2011 & Hospital & 420 & 29.1 & 17.1 & 14.5 & 6.8 & $\underline{13.3}$ & 14.8 \\
\hline Shenzhen [44] & 2009 & Hospital & 589 & 38.0 & & 9.3 & & 6.8 & 47.3 \\
\hline Chongqing [45] & $2011-2013$ & Hospital & 1976 & & & 10.5 & & & 24.5 \\
\hline Fujian [46] & 2010-2011 & Survey & 1389 & & & 5.4 & & 2.3 & 25.3 \\
\hline
\end{tabular}

${ }^{\mathrm{a}} \mathrm{Mtb}$ M. tuberculosis, MDR multidrug resistance, CDC Chinese Centre for Disease Control and Prevention, FQs fluoroquinolones

${ }^{b}$ Data was derived based on sampling sources of hospital (Hospital), local TB control system (CDC) or nationwide random survey

${ }^{\mathrm{C}}$ The data presented the resistance rate of FQ-resistant from total or from MDR Mtb isolates examined (resistant to Ofloxacin, in Italian; resistant to levofloxacin, underlined)

The present investigation included data from a designated TB hospital in Heilongjiang Province from 2007 through 2012, with rates of any first-line drug resistance and MDR-TB of 57.0 and $22.8 \%$, respectively, whereas these rates were 48.7 and $19.3 \%$, respectively, in 2012 . The rate of drug resistance in this area was highest in 2009 and decreased in 2010 and later. In the investigated hospital, the DST availability was lower than $20 \%$ before 2009, when DST was performed only if patients suffer treatment failure or no obvious effect of treatment. Since 2010, DST became more frequent for culture-positive TB patients. The increased proportion of DST might be the reason for the decrease of drug resistance rates from 2009 to 2010 . However, DST is still in only about $50 \%$ of the culture-positive TB cases now. This inadequate DST availability may maintain the comparatively high prevalence of MDR TB.

Primary resistance in this area is also an important issue. Of the new cases investigated in 2012, approximately $40 \%$ were resistant to any first-line drug, whereas $18.5 \%$ were resistant to either isoniazid or rifampin, a population at risk of developing MDR, and $8.9 \%$ were MDR-TB. Notably, approximately $2 \%$ of new cases were resistant to all of the 4 first-line drugs (pan-resistance). This TB population is the potential resource of the XDR-TB if the treatment strategy is not appropriate. Regarding China's TB treatment regulations $[26,48]$, all new pulmonary $\mathrm{TB}$ cases are treated free of charge with first-line drugs if DST is not available.

Furthermore, less than $20 \%$ of the TB medical facilities that use second-line drugs for MDR-TB treatment design therapy regimens based on DST [34]. This situation will cause the appearance of additional MDR and XDR M. tuberculosis cases.

In the new cases, high proportions with cavity (approximately $60 \%$ ) and short hospitalization (19 \%) should also be identified. These patients are released from hospitals after the initial treatment and may be 
a highly dangerous source of infection for susceptible individuals, causing the spread of both $\mathrm{TB}$ and MDR TB. It is important to decrease the proportion of non-standard hospitalization and appropriately treat the cases with cavity to achieve the successful control of primary TB spread.

The resistance situation to FQs distributed variously in different TB populations (outpatients and inpatients) and in different areas of China, with a higher resistance rate in hospitalized patients. In addition, the MDR strains would be more likely to resistant to FQs $[10,34$, $38,43-47]$.

In recent years, levofloxacin, the most frequently used fluoroquinolone in China, has become the first choice fluoroquinolone for MDR-TB treatment, and moxifloxacin is used as an alternative fluoroquinolone based on China's clinical practice guidelines for TB [26].

Based on the present data, there is a limited choice of fluoroquinolones for the ideal regimen for MDR-TB therapy because approximately $15-17 \%$ and $14-15 \%$ of the MDR-TB cases are not susceptible to the 3 and 4 of fluoroquinolones, respectively. Importantly, at least $13 \%$ of the MDR M. tuberculosis isolates were not susceptible to any of the 5 fluoroquinolones. The high prevalence of drug resistance may become uncontrolled if measures are not effective and may indicate an increase of XDR-TB.

MDR is an independent risk factor associated with resistance to fluoroquinolones [9]. The high rate of MDRTB in the investigated area $(22.8 \%)$ is a related causative reason for the high prevalence of fluoroquinolone resistance. Furthermore, the fluoroquinolones are commonly used antibiotics for clinical departments, and antibiotics abuse has not been well controlled in China [11-14]. Controlling the resistance to fluoroquinolones may be achieved through the proper management of MDR-TB cases and the proper use of such antibiotics.

\section{Conclusions}

The results of our study indicate that there was a high prevalence of drug resistance to the first-line drugs and multidrug resistance (MDR) in Heilongjiang Province, northeastern China. Among MDR TB, more than $10 \%$ was resistant to fluoroquinolones, indicating a severe second line drug resistance. After analysis of the risks of MDR-TB, we should pay more attention to patients' age, re-treatment proportion, cavity lesion, and high proportion of shorter hospitalization to get control tuberculosis more efficiently.

\section{Abbreviations}

DOTS: Directly observed treatment short course; DST: Drug susceptibility testing; MDR: Multidrug resistant; MGIT: Mycobacterium Growth Indicator Tube; MICs: Minimum inhibition concentrations; TB: Tuberculosis; WHO: World Health Organization; XDR: Extensively drug-resistant

\section{Acknowledgments}

We thank Nakajima at Hokkaido University, Japan, for critical review and editorial assistance during manuscript preparation. This work was supported by the Grant for Creation Program of Heilongjiang Province to J-L.W (YJSCX2012$233 \mathrm{HLJ}$ ) and by the Grant for Innovation Team Program of Heilongjiang Province to $H$. L and D. L.

\section{Funding}

The Grant for Creation Program of Heilongjiang Province to J-L.W and the Grant for Innovation Team Program of Heilongjiang Province to $H$. $L$ and D. L. supported this project.

\section{Availability of data and materials}

All the data supporting the findings are contained within the manuscript.

\section{Authors' contribution}

Conceived the investigation: $\mathrm{HL}$; designed the investigation: $\mathrm{HL}, \mathrm{DL}, \mathrm{B}-\mathrm{YJ}$; performed the clinical experiments including MGIT 960 culture and DST, PNB/TCH tests, DST to FQs: L-NZ, C-LZ; performed sample preparation for molecular identification: C-LF, L-NZ, C-LZ; performed DNA extraction: C-LF, J-YC, C-XS; performed PCR identification of isolates and Beijing family: J-YC, Y-PM; performed MIC assay: J-LW, Y-PM; stored and recovered clinical isolates: $C-L F, C-L Z$; analyzed the data: $X-L P, D L, H L, T H, J-L W$; collected clinical records: B-YJ, J-YC, X-LP, C-XS, C-BD; statistical analysis: L-ZZ, J-LW; wrote the paper: DL, $J-L W, X-L P, H L$. All authors read and approved the final manuscript.

\section{Competing interests}

The authors declare that they have no competing interests.

\section{Consent for publication}

Not applicable.

\section{Ethics approval and consent to participate}

The study was approved by the Institutional Research Board of the University of Harbin Medical University (Ethics Reference No.: HMUIRB20160001).

\section{Author details}

${ }^{1}$ Department of Microbiology; Wu Lien-Teh Institute, Harbin Medical University, Harbin, China. ${ }^{2}$ Heilongjiang Provincial Key Laboratory of Infection and Immunity, Harbin, China. ${ }^{3}$ Department of Parasitology, Harbin Medical University; Key Laboratory of Pathogen Biology, Harbin, China. ${ }^{4}$ Harbin Chest Hospital, Harbin, China. ${ }^{5}$ Shen Zhen Protection and Treatment Center for Occupational Diseases, Shenzhen, China. ${ }^{6}$ Division of Disaster-related Infectious Diseases, International Research Institute of Disaster Science, Tohoku University, Sendai, Japan.

Received: 27 November 2015 Accepted: 17 September 2016 Published online: 27 September 2016

\section{References}

1. Zhao Y, Xu S, Wang L, Chin DP, Wang S, Jiang G, et al. National survey of drug-resistant tuberculosis in China. N Engl J Med. 2012;366:2161-70.

2. He GX, Zhao YL, Jiang GL, Liu YH, Xia H, Wang SF, et al. Prevalence of tuberculosis drug resistance in 10 provinces of China. BMC Infect Dis. 2008; $8: 166$

3. Xie YG, Li FB, Yan XL. WHO TB drug resistant survey in Heilongjiang province [in Chinese]. J Chin Antituberculosis Assoc. 2008;30:395-98.

4. He GX, Xie YG, Wang LX, Borgdorff MW, van der Werf MJ, Fan JH, et al. Follow-up of patients with multidrug resistant tuberculosis four years after standardized first-line drug treatment. PLoS One. 2010;5:e10799.

5. Shen X, DeRiemer K, Yuan ZA, Shen M, Xia Z, Gui X, et al. Drug-resistant tuberculosis in Shanghai, China, 2000-2006: prevalence, trends and risk factors. Int J Tuberc Lung Dis. 2009;13:253-9.

6. Otero L, Krapp F, Tomatis C, Zamudio C, Matthys F, Gotuzzo E, et al. High prevalence of primary multidrug resistant tuberculosis in persons with no known risk factors. PLoS One. 2011;6:e26276.

7. Faustini A, Hall AJ, Perucci CA. Risk factors for multidrug resistant tuberculosis in Europe: a systematic review. Thorax. 2006;61:158-63.

8. Law WS, Yew WW, Chiu Leung C, Kam KM, Tam CM, Chan CK, et al. Risk factors for multidrug-resistant tuberculosis in Hong Kong. Int J Tuberc Lung Dis. 2008;12:1065-70. 
9. Wang JY, Lee LN, Lai HC, Wang SK, Jan IS, Yu CJ, et al. Fluoroquinolone resistance in Mycobacterium tuberculosis isolates: associated genetic mutations and relationship to antimicrobial exposure. J Antimicrob Chemother. 2007:59:860-5.

10. Hu Y, Hoffner S, Wu L, Zhao Q, Jiang W, Xu B. Prevalence and genetic characterization of second-line drug-resistant and extensively drug-resistant Mycobacterium tuberculosis in Rural China. Antimicrob Agents Chemother. 2013;57:3857-63.

11. Wu AH, Li CH, Wen XM, Ren N. Nationl healthcare-associated infection surveillance system point prevalence survey of antimcrobial use in 740 Chinese hospitals in 2010 [in Chinese]. Chin J Infect Control. 2012;11:7-11.

12. Jia HY. Survey on using condition of antibiotics in inpatients [in Chinese]. Mod Med J China. 2012;14:63-7.

13. Chen $\mathrm{HQ}$, Zhang L. Clinical application of quinolones in pediatric infectious diseases [in Chinese]. Clin Med Eng. 2011;18:795-96.

14. Zhang YD, Zong JS, Sun YX, Pan LZ, Hu ZY, Liang XF, et al. Cross-sectional study on nosocomial infections and use of antibiotics in hospitalized patients [in Chinese]. Chin J Nosocomiol. 2012;22:2165-68.

15. Jeon CY, Hwang SH, Min JH, Prevots DR, Goldfeder LC, Lee H, et al. Extensively drug-resistant tuberculosis in South Korea: risk factors and treatment outcomes among patients at a tertiary referral hospital. Clin Infect Dis. 2008;46:42-9.

16. Dalton T, Cegielski P, Akksilp S, Asencios L, Campos Caoili J, Cho SN, et al. Prevalence of and risk factors for resistance to second-line drugs in people with multidrug-resistant tuberculosis in eight countries: a prospective cohort study. Lancet. 2012;380:1406-17.

17. Coban AY, Birinci A, Ekinci B, Durupinar B. Drug susceptibility testing of Mycobacterium tuberculosis by the broth microdilution method with $7 \mathrm{H} 9$ broth. Mem Inst Oswaldo Cruz. 2004;99:111-3.

18. Cheng AF, Yew WW, Chan EW, Chin ML, Hui MM, Chan RC. Multiplex PCR amplimer conformation analysis for rapid detection of gyrA mutations in fluoroquinolone-resistant Mycobacterium tuberculosis clinical isolates. Antimicrob Agents Chemother. 2004;48:596-601.

19. Cui Z, Wang J, Lu J, Huang X, Hu Z. Association of mutation patterns in gyrA/B genes and ofloxacin resistance levels in Mycobacterium tuberculosis isolates from East China in 2009. BMC Infect Dis. 2011:11:78.

20. Pranger AD, Alffenaar JW, Aarnoutse RE. Fluoroquinolones, the cornerstone of treatment of drug-resistant tuberculosis: a pharmacokinetic and pharmacodynamic approach. Curr Pharm Des. 2011;17:2900-30.

21. Wang SM. Protocols of Laboratory examinations for Tuberculosis Diagnosis, 1st ed. Beijing: Cultural Education Press; 2006.

22. Elbir H, Abdel-Muhsin AM, Babiker A. A one-step DNA PCR-based method for the detection of Mycobacterium tuberculosis complex grown on Lowenstein-Jensen media. Am J Trop Med Hyg. 2008;78:316-7.

23. van Soolingen D, Hermans PW, de Haas PE, Soll DR, van Embden JD. Occurrence and stability of insertion sequences in Mycobacterium tuberculosis complex strains: evaluation of an insertion sequencedependent DNA polymorphism as a tool in the epidemiology of tuberculosis. J Clin Microbiol. 1991;29:2578-86.

24. Wang J, Liu Y, Zhang CL, Ji BY, Zhang LZ, Shao YZ, et al. Genotypes and characteristics of clustering and drug susceptibility of Mycobacterium tuberculosis isolates collected in Heilongjiang Province, China. J Clin Microbiol. 2011:49:1354-62.

25. Huard RC, Lazzarini LC, Butler WR, van Soolingen D, Ho JL. PCR-based method to differentiate the subspecies of the Mycobacterium tuberculosis complex on the basis of genomic deletions. J Clin Microbiol. 2003;41:1637-50.

26. Wang XY. The study of the trend of drug resistance of 1011 mycobacterium tuberculosis [in Chinese]. Lab Med Clin. 2010;7:712-13.

27. Jiao WW, Liu ZG, Han R, Zhao XQ, Dong F, Dong HY, et al. Prevalence of drug resistant Mycobacterium tuberculosis among children in China. Tuberculosis. 2015;95:315-20.

28. Zhang Z, Lu J, Wang Y, Pang Y, Zhao Y. Prevalence and molecular characterization of fluoroquinolone-resistant Mycobacterium tuberculosis isolates in China. Antimicrob Agents Chemother. 2014;58:364-9.

29. Yuan YL, Yang XJ, Yang GX, Li XK, Wang B, Zhang WY, et al. Survillance of Mycobacteria tuberculosis drug resistance in Jilin Province [in Chinese]. Chin J of Public Health Eng. 2015;14:70-5.

30. Wang XP, Wang XL, Xiao HX, Wang FR, Liu XN, Shen YC, et al. Analysis of drug resistance of Mycobactria tuberculosis survillance in Ningxia Province [in Chinese]. Ningxia Med J. 2015;37:78-80.
31. Cheng X, Li YF, Rao Y, Yang XH, Du H. Drug resistance situation in 2271 tuberculosis patients and epidemiological characteristics analysis [in Chinese]. Chongqing Med. 2015;44:1635-37.

32. Liu Q, Zhu L, Shao Y, Song H, Li G, Zhou Y, et al. Rates and risk factors for drug resistance tuberculosis in Northeastern China. BMC Public Health. 2013;13:1171.

33. Chen YY, Chang JR, Kuo SC, Tseng FC, Huang WC, Huang TS, et al. Molecular epidemiology of tuberculosis in Kaohsiung City located at southern Taiwan, 2000-2008. PLoS One. 2015;10:e0117061.

34. Wang D, Yang C, Kuang T, Lei H, Meng X, Tong A, et al. Prevalence of multidrug and extensively drug-resistant tuberculosis in Beijing, China: a hospital-based retrospective study. Jpn J Infect Dis. 2010;63:368-71.

35. Qi YC, Ma MJ, Li DJ, Chen MJ, Lu QB, Li XJ, et al. Multidrug-resistant and extensively drug-resistant tuberculosis in multi-ethnic region, Xinjiang Uygur Autonomous Region, China. PLoS One. 2012;7:e32103.

36. Wang XH, Ma AG, Han XX, Gu XM, Fu LP, Li PG, et al. Correlations between drug resistance of Beijing/W lineage clinical isolates of Mycobacterium tuberculosis and sublineages: a 2009-2013 prospective study in Xinjiang province, China. Med Sci Monit. 2015;21:1313-8.

37. Chen LY, Ma L, Ru HH, Wang JY, Xu L. Analysis of drug resistance of 279 mycobacterium tuberculosis strains [in Chinese]. J Kunming Med Univ. 2014; $35: 4$.

38. Zhao LL, Chen $Y$, Chen ZN, Liu HC, Hu PL, Sun Q, et al. Prevalence and molecular characteristics of drug-resistant Mycobacterium tuberculosis in Hunan, China. Antimicrob Agents Chemother. 2014;58:3475-80.

39. Pang H, Tong J, Liu HC, Du YG, Zhao XQ, Yi J, et al. Molecular characterization and drug-resistance of Mycobacterium tuberculosis strains in Xuzhou, China. Biomed Environ Sci. 2014;27:960-4.

40. Xia Q, Zhao LL, Li F, Fan YM, Chen YY, Wu BB, et al. Phenotypic and genotypic characterization of pyrazinamide resistance among multidrugresistant Mycobacterium tuberculosis isolates in Zhejiang, China. Antimicrob Agents Chemother. 2015;59:1690-5

41. Yuan X, Zhang T, Kawakami K, Zhu J, Zheng W, Li H, et al. Genotyping and clinical characteristics of multidrug and extensively drug-resistant tuberculosis in a tertiary care tuberculosis hospital in China. BMC Infect Dis. 2013;13:315

42. Yang $Y$, Zhou $C$, Shi L, Meng H, Yan $H$. Prevalence and characterization of drug-resistant tuberculosis in a local hospital of Northeast China. Int J Infect Dis. 2014:22:83-6.

43. Xu DF, Wang Q, Li Z, Li DF. Resistance analysis of the first and second line anti-Tb drugs in 420 clinical Mycobacteria tuberculosis isolates from Anhui Province [in Chinese]. Chin J Zoonoses. 2014:30:54-7.

44. Liu HM, Zhan NY, Chen JB, Xiao YY, Luo K, Wu C, et al. Analysis of drug resistance of 589 Mycobacterium Tuberculosis strains for 10 type antituberculosis drugs [in Chinese]. J Clin Pulm Med. 2011;16:557-58.

45. Zhang D, Liu B, Wang Y, Pang Y. Rapid molecular screening for multidrugresistant tuberculosis in a resource-limited region of China. Trop Med Int Health. 2014:19:1259-66

46. Chen Q, Pang Y, Liang Q, Lin S, Wang Y, Lin J, et al. Molecular characteristics of MDR Mycobacterium tuberculosis strains isolated in Fujian, China. Tuberculosis (Edinb). 2014;94:159-61.

47. Yan LP, Xiao HP. Clinical monitor and analysis on Ofloxacin resistance of pulmonary tuberculosis [in Chinese]. Chin J Antituberculosis. 2009;31:477-80.

48. Xiao HP. Guideline for chemmotherapy of drug-resistant tuberculosis [In Chinese]. 1 stth ed. Beijing: People's Medical Publishing House; 2010.

\section{Submit your next manuscript to BioMed Central and we will help you at every step:}

- We accept pre-submission inquiries

- Our selector tool helps you to find the most relevant journal

- We provide round the clock customer support

- Convenient online submission

- Thorough peer review

- Inclusion in PubMed and all major indexing services

- Maximum visibility for your research

Submit your manuscript at www.biomedcentral.com/submit 